\title{
Smoking prevalence and associated risk factors among healthcare professionals in Nicosia general hospital, Cyprus: a cross-sectional study
}

Stavri Zinonos ${ }^{1 *}$, Theodora Zachariadou ${ }^{2}$, Savvas Zannetos ${ }^{2}$, Andrie G. Panayiotou ${ }^{3}$ and Andreas Georgiou ${ }^{4}$

\begin{abstract}
Background: In recent years, a significant progress has been achieved globally in reduction of smoking among physicians and nurses, however, in some countries the smoking prevalence of health professionals is maintained at very high levels, without significant difference from the general population. This study aims to investigate the prevalence of smoking among physicians and nurses working at Nicosia General Hospital, as well as their knowledge and attitudes towards smoking cessation strategies.

Methods: This is a cross-sectional questionnaire-based study. The study consisted of 119 doctors and 392 nurses currently working at Nicosia General Hospital in Cyprus. Study participants were recruited from all hospital wards between May and June 2008. Both physicians and nurses were asked to answer an anonymous questionnaire, which included questions regarding their smoking habits, knowledge and attitudes about smoking and smoking cessation strategies.

Results: Overall smoking prevalence among healthcare professionals was $28.2 \%$ (28.6\% among physicians and $28.1 \%$ among nurses). Multivariate analysis revealed that being male, younger than 34 years old, unmarried and with a family history of smoking were associated with increased likelihood of being a current smoker. An impressive $72 \%$ of current smokers reported that they wished to quit smoking, however, only $5.6 \%$ of physicians and $6.9 \%$ of nurses, reported ever using any smoking cessation aids. Never- smokers counseled their patients to quit smoking more often ( $96.4 \%)$ compared to former $(84.6 \%)$ and current smokers $(72.7 \%),(p<0.001)$. In addition, those who felt more confident about their knowledge regarding smoking cessation, reported counseling their patients to quit smoking more often compared to those who did not ( $92 \%$ vs $60 \%, p<0.001)$.

Conclusions: Smoking prevalence among physicians and nurses working at Nicosia General Hospital was similar to that of the general Cypriot population. Further training of healthcare professionals towards smoking cessation strategies is needed in order to improve their knowledge and consequently their efforts on counseling and support to their patients who wish to quit smoking.
\end{abstract}

Keywords: Smoking prevalence, Healthcare professionals, Smoking attitudes

\footnotetext{
*Correspondence: zinonos.stavri@ucy.ac.cy

${ }^{1}$ Health Center of University of Cyprus, Cyprus International Institute for

Environmental and Public Health in association with the Harvard T.H. Chan

School of Public Health, Cyprus University of Technology, 1, Panepistimiou

Avenue, 2109 Aglantzia, Nicosia, Cyprus

Full list of author information is available at the end of the article
} 


\section{Background}

Tobacco use has been generally acknowledged as one of the biggest public health threats ever faced, with more than one billion smokers worldwide [1]. According to the World Health Organization (WHO), smoking kills nearly 6 million people annually, an average of one person every six seconds, and accounts for 6 and $12 \%$ of all female and male deaths respectively $[1,2]$. In addition, in 2014, World Health Organization after the revision of relevant data regarding smoking, reports that tobacco use is the direct cause of more than five million deaths, while more than 600,000 deaths are attributable to second-hand smoking [1]. If current trends continue and drastic measures are not taken to control tobacco use, annual tobacco deaths are expected to rise to 10 million worldwide by 2030 [2]. The biggest impact of smoking is through noncommunicable diseases, although it may also increase the risk of communicable diseases such as tuberculosis and lower tract respiratory infections [3]. It is responsible for $87 \%$ of all lung cancer deaths [4], $42 \%$ of chronic respiratory diseases and nearly $10 \%$ of cardiovascular diseases [5].

Healthcare professionals should be active in smoking prevention, as part of their daily practice [6]. They should discuss with their patients the harmful effects of smoking and available smoking cessation interventions and support them through any smoking cessation process [7-11]. There is evidence that when physicians and nurses offer specific assistance and appropriate support, a large percentage of their patients who smoke will try to quit, even those with low motivation to quit [12]. Furthermore, the smoking status of healthcare professionals appears to be a critically important determinant of their ability to assist their patients to control tobacco use. Studies suggest that as smoking rates among physicians decline, a similar reduction will be observed in the general population as well [13]. However, while in most developed countries the rate of physicians and nurses who smoke has declined significantly over the last 30 years [14], in other countries it has been maintained at very high levels and without a significant difference to that of the general population [14].

In Cyprus, smoking prevalence among the general population is $26.5 \%$ [9] and so far, no studies have been performed regarding the prevalence of smoking among physicians and nurses. This study aims to estimate smoking prevalence among physicians and nurses working at Nicosia General Hospital (NGH), the largest hospital in Cyprus and to investigate their knowledge and attitudes towards smoking and smoking cessation strategies, as well as possible interactions with their own smoking status.

\section{Methods}

\section{Study population}

This cross-sectional study included 119 doctors and 392 nurses working at NGH, in Cyprus. Study participants were recruited from all hospital wards between May and June 2008. A self - completed anonymous questionnaire was administered to all participants after signing a consent form. The NGH is the largest hospital of the island providing tertiary care and is the referral hospital of all other hospitals and clinics of the island. Therefore, the study sample could be considered representative of public sector physicians and nurses.

\section{Sample size calculations and recruitment}

All physicians working at NGH were asked to participate in the survey $(n=175)$ [15]. Twenty-nine of them were absent at the time of the survey. Further, 27 refused to participate, thus the response rate for physicians was $81.5 \%$.

For nurses, a sample size calculation was performed as follows. For a hypothesized smoking prevalence of $26 \%$ (population average), with a Confidence Level of $95 \%$, a margin of error of $\pm 5 \%$ and no Finite Population Correction application, a sample of 296 nurses was needed. A conservative $40 \%$ non-response rate, due to no prior experience on similar studies, was added, giving a total sample size of 493 nurses. From the total of 852 [16] nurses working at NGH, 493 were randomly selected among all wards of the hospital using a hospital payroll list with a unique consecutive number attached to each name and the random sample command in SPSS was used to select the appropriate random sample. At the time, 23 nurses were absent and did not participate. Out of the 493 randomly selected nurses, 392 agreed to participate giving a response rate of $79.5 \%$.

\section{Questionnaires}

Both physicians and nurses were sought out on a oneto-one basis by the study researchers and asked to answer an anonymous questionnaire, which was taken from a Greek study by Sotiropoulos et al. [7]. The questionnaire included questions regarding smoking history, attitudes about smoking and smoking cessation as well as the demographic characteristics of the participants.

Regarding smoking status, respondents were classified as current smokers, former smokers and never-smokers. Participants were asked to state how often they counsel patients to stop smoking (rarely/never/sometimes/often), about their attitude as to whether healthcare professionals should undergo specific training to help their patients who wish to quit smoking (Yes/No) and about their attitude as to whether smoking should be prohibited in hospitals and in public places (Yes/No). 
Nicotine Dependence was evaluated with the use of the Fagerstrom Nicotine Tolerance Questionnaire (FTQ) [17]. Smokers were divided into three categories: low nicotine dependence, medium nicotine dependence and high nicotine dependence following the recommended procedure and cut off points from the creators of the questionnaire.

\section{Definitions}

As current smokers were defined those who reported smoking at least 100 cigarettes in their lifetime and who, at the time of survey, smoked either every day or some days. As former smokers were defined those who reported smoking at least 100 cigarettes in their lifetime and who, at the time of the survey, did not smoke at all. Respondents who reported never having smoked 100 cigarettes, were defined as never smokers [18]. The family history of smoking was determined with the question "Does anyone else smoke in the family?"

On the basis of the FTQ, smokers were divided into low nicotine dependent category $(0 \leq \mathrm{FTQ}$ score $\leq 3)$, medium nicotine dependent $(4 \leq$ FTQ score $\leq 6)$ and high nicotine dependent ( $\leq$ FTQ score $\leq 10)$ [17]. Packyears of smoking were defined by multiplying the number of cigarettes smoked per day by the number of years the person has smoked and then divided by 20 (cigarettes of one pack).

\section{Statistical analysis}

Continuous variables were expressed as medians (after checking their distribution), while nominal variables were expressed as frequencies and percentages. Chisquare test or Fisher's exact test, were used to examine the association between variables. Moreover, MannWhitney test was used to examine the statistical relationship among non-normally distributed data. A multiple forward stepwise logistic regression analysis was used to determine independent predictors of the likelihood of being current smoker vs. never - smokers. The covariates examined were gender, age, marital status, having children and family history of smoking. Indicator variables were created for all categorical variables and odds ratios with $95 \%$ confidence intervals (CI) were presented. Level of statistical significance was defined at $\alpha=0.05$ therefore, $\mathrm{p}$ value $<0.05$ was required for a covariate to be in the model. No interaction effects between covariates were statistical significant, thus not included in the model. In addition, for the logistic regression, due to small sample size of the variable marital status, the categories unmarried, divorced and widowed were combines into one group.

All statistical analysis was performed using the IBM SPSS Statistics Software, version 20.0.

\section{Results}

\section{Characteristics of the study population}

From the total 511 participants, 119 were physicians (23.3\%) and 392 were nurses (76.7 \%). The participants aged 25-34 years old represented $41.7 \%$ of the study population. Sixty three percent of physicians and $68.9 \%$ of nurses were married. Detailed characteristics of the study population are presented in Table 1.

\section{Smoking prevalence and factors associated with smoking status}

The overall prevalence of smoking among healthcare professionals working at $\mathrm{NGH}$ was $28.2 \%$ and it was similar among physicians and nurses (28.6 and $28.1 \%$ respectively, $p=0.874)$. A total of 186 participants were current or former smokers (36.4\%). Among all, $16 \%$ of doctors and $5.9 \%$ of nurses were former smokers, whereas $55.5 \%$ of physicians and $66.1 \%$ of nurses had never used tobacco in their life. Moreover, $8 \%$ of nurses and $5.9 \%$ of physicians admitted that they smoke in front of their patients.

Factors associated univariately with smoking status were: younger age $(p<0.001)$, being male $(p<0.001)$, being single $(p=0.002)$ and having a family history of smoking $(p<0.001)$. Details are shown in Table 2.

In addition, classification analysis was performed. Several algorithms (e.g., random forests, naïve Bayes) were implemented with the best (in terms of accuracy) performing algorithm to be logistic regression. Thus, in a multiple forward stepwise logistic regression model, with smoking status (current vs. never- smokers) as the dependent variable, the final fitted model included gender $(p<0.001)$, age $(p=0.004)$, marital status $(p=0.012)$ and family history of smoking $(p<0.001)$. Thus, male, unmarried, younger than 34 years old healthcare professionals with family history of smoking were the most likely to be smokers. Details are presented in Table 3.

\section{Smoking cessation attitudes among current smokers}

Out of 144 current smokers, $71.8 \%$ reported that they would like to quit. Almost half of physicians (48.5\%) and nurses (52\%) who smoke have attempted to quit smoking in the past; however, only $5.6 \%$ of physicians and $6.9 \%$ of nurses reported using any smoking cessation aids. The attempt to quit smoking was successful for more than half of the physicians $(62.5 \%)$ compared to only $31.5 \%$ of nurses $(p=0.025)$.

Univariate analysis revealed that nurses and doctors advised their patients to quit smoking at similar rates ( $89.6 \%$ vs. $80.7 \%$ respectively; $p=0.089)$. No statistically significant difference was found between physicians and nurses who rarely or never counsel their patients to quit smoking (8.4 \% vs. $4.5 \% ; p=0.089)$. 
Table 1 Characteristics of the study population $(n=511)$

\begin{tabular}{|c|c|c|c|c|c|c|c|}
\hline & \multicolumn{2}{|l|}{ All } & \multicolumn{2}{|c|}{ Physicians } & \multicolumn{2}{|c|}{ Nurses } & \multirow[t]{2}{*}{$p$ value } \\
\hline & $\mathrm{N}$ & $\%$ & $\mathrm{~N}$ & $\%$ & $\mathrm{~N}$ & $\%$ & \\
\hline \multicolumn{8}{|l|}{ Sex } \\
\hline Male & 139 & 27.2 & 71 & 59.7 & 68 & 17.3 & \multirow[t]{2}{*}{$<0.001$} \\
\hline Female & 372 & 72.8 & 48 & 40.3 & 324 & 82.7 & \\
\hline \multicolumn{8}{|l|}{ Age (years) } \\
\hline $25-34$ & 213 & 41.7 & 48 & 40.3 & 165 & 42.1 & \multirow[t]{4}{*}{0.925} \\
\hline $35-44$ & 111 & 21.7 & 28 & 23.5 & 83 & 21.2 & \\
\hline $45-54$ & 135 & 26.4 & 30 & 25.2 & 105 & 26.8 & \\
\hline $55-64$ & 52 & 10.2 & 13 & 10.9 & 39 & 9.9 & \\
\hline \multicolumn{8}{|c|}{ Does anyone else smoke in the family? } \\
\hline Yes & 99 & 19.4 & 30 & 25.2 & 69 & 17.6 & \multirow[t]{2}{*}{0.066} \\
\hline No & 412 & 80.6 & 89 & 74.8 & 323 & 82.4 & \\
\hline \multicolumn{8}{|l|}{ Smoking Status } \\
\hline Current & 144 & 28.2 & 34 & 28.6 & 110 & 28.1 & \multirow[t]{3}{*}{0.002} \\
\hline Former & 42 & 8.2 & 19 & 16.0 & 23 & 5.9 & \\
\hline Never & 325 & 63.6 & 66 & 55.5 & 259 & 66.1 & \\
\hline \multicolumn{8}{|l|}{ FTQ-Level of Dependence } \\
\hline Low nicotine dependent & 87 & 61.3 & 20 & 58.8 & 67 & 62.0 & \multirow[t]{3}{*}{0.385} \\
\hline Medium nicotine dependent & 39 & 27.5 & 8 & 23.5 & 31 & 28.7 & \\
\hline High nicotine dependent & 16 & 11.3 & 6 & 17.6 & 10 & 9.3 & \\
\hline Pack- years of smoking & \multicolumn{2}{|c|}{ Median(IQR) } & \multicolumn{2}{|c|}{ Median(IQR) } & \multicolumn{2}{|c|}{ Median(IQR) } & $p$ value ${ }^{*}$ \\
\hline Current smokers & \multicolumn{2}{|c|}{$7.5(10.50)$} & \multicolumn{2}{|c|}{$8.5(10.25)$} & \multicolumn{2}{|c|}{$7.50(10.50)$} & 0.857 \\
\hline Former smokers & \multicolumn{2}{|c|}{$5.0(8.50)$} & \multicolumn{2}{|c|}{$5.0(18.65)$} & \multicolumn{2}{|c|}{$1.0(5.50)$} & 0.061 \\
\hline
\end{tabular}

"Mann Whitney test was used

Table 2 Characteristics of the study population by smoking status

\begin{tabular}{|c|c|c|c|c|c|c|c|}
\hline & \multicolumn{2}{|c|}{ Never Smokers } & \multicolumn{2}{|c|}{ Current Smokers } & \multicolumn{2}{|c|}{ Former Smokers } & \multirow[t]{2}{*}{$p$ value } \\
\hline & $\mathrm{N}$ & $\%$ & $\bar{N}$ & $\%$ & $\bar{N}$ & $\%$ & \\
\hline \multicolumn{8}{|l|}{ Sex } \\
\hline Male & 64 & 46 & 56 & 40.3 & 19 & 13.7 & \multirow[t]{2}{*}{$<0.001$} \\
\hline Female & 261 & 70.2 & 88 & 23.7 & 23 & 6.2 & \\
\hline \multicolumn{8}{|l|}{ Age (years) } \\
\hline $25-34$ & 122 & 57.3 & 78 & 36.6 & 13 & 6.1 & \multirow[t]{4}{*}{$<0.001$} \\
\hline $35-44$ & 91 & 82.0 & 15 & 13.5 & 5 & 4.5 & \\
\hline $45-54$ & 79 & 58.5 & 39 & 28.9 & 17 & 12.6 & \\
\hline $55-64$ & 33 & 63.5 & 12 & 23.1 & 7 & 13.5 & \\
\hline \multicolumn{8}{|l|}{ Marital Status } \\
\hline Unmarried & 73 & 53.3 & 54 & 39.4 & 10 & 7.3 & \multirow[t]{4}{*}{$0.002^{*}$} \\
\hline Married & 239 & 69.3 & 77 & 22.3 & 29 & 8.4 & \\
\hline Divorced & 8 & 42.1 & 9 & 47.4 & 2 & 10.5 & \\
\hline Widowed & 5 & 50 & 4 & 40 & 1 & 10 & \\
\hline \multicolumn{8}{|c|}{ Does anyone else smoke in the family? } \\
\hline Yes & 13 & 13.1 & 78 & 78.8 & 8 & 8.1 & \multirow[t]{2}{*}{$<0.001$} \\
\hline No & 312 & 75.7 & 66 & 16 & 34 & 8.3 & \\
\hline
\end{tabular}


Table 3 Multivariable logistic regression of smoking status against demographic characteristics

\begin{tabular}{|c|c|c|c|c|c|c|c|c|c|c|}
\hline \multicolumn{6}{|l|}{ Unadjusted } & \multicolumn{5}{|c|}{ Adjusted } \\
\hline & \multirow{2}{*}{$\begin{array}{l}\text { B } \\
-1.087\end{array}$} & $\begin{array}{l}p \text { value } \\
.000\end{array}$ & $\begin{array}{l}\text { Odds ratio } \\
.34\end{array}$ & \multicolumn{2}{|c|}{$\begin{array}{l}95 \% \text { C.I. for } \\
\text { odds ratio }\end{array}$} & $\frac{B}{-1.146}$ & $\begin{array}{l}p \text { value } \\
.000\end{array}$ & $\begin{array}{l}\text { Odds ratio } \\
0.32\end{array}$ & \multicolumn{2}{|c|}{$\begin{array}{l}95 \% \text { C.I. for } \\
\text { odds ratio }\end{array}$} \\
\hline \multicolumn{10}{|l|}{ Gender } & \\
\hline Male & .954 & .000 & 2.60 & 1.68 & 4.00 & 1.012 & .000 & 2.75 & 1.59 & 4.75 \\
\hline \multicolumn{11}{|l|}{ Age (years) } \\
\hline $35-44$ & & & & & & -1.206 & .004 & 0.30 & 0.13 & 0.69 \\
\hline $45-54$ & & & & & & .005 & .989 & 1.00 & 0.51 & 1.99 \\
\hline $55-64$ & & & & & & -.376 & .447 & 0.69 & 0.26 & 1.81 \\
\hline \multicolumn{11}{|c|}{ Marital Status } \\
\hline Married $^{a}$ & & & & & & -.769 & .012 & 0.46 & 0.25 & 0.85 \\
\hline \multicolumn{11}{|c|}{ Does anyone else smoke in the family? } \\
\hline Yes & & & & & & 1.109 & 0.000 & 3.03 & 1.51 & 6.10 \\
\hline
\end{tabular}

${ }^{a}$ compared to unmarried/widowed/divorced as a combined category

Details about healthcare professionals' attitudes towards counseling patients to quit smoking, by smoking status and level of dependence are presented in Table 4. No statistical significant association was found between the level of nicotine dependence and attitudes on smoking prohibition in hospitals $(p=0.951)$ or public places $(p=0.287)$.

Although the majority of physicians and nurses believed that they had the necessary knowledge to counsel patients on how to quit smoking ( $85.7 \%$ vs $92.6 \%$ respectively, $p=0.052$ ), those (doctors and nurses) who felt more confident about their knowledge regarding smoking cessation, reported counseling their patients to quit smoking more often compared to those who did not (92\% vs $60 \%, p<0.001)$.

\section{Discussion}

The present study examined the smoking behavior among Cypriot physicians and nurses working in $\mathrm{NGH}$, which is the largest tertiary hospital of Cyprus. Smoking prevalence among physicians and nurses was slightly higher (28.6 and $28.1 \%)$ compared to that of the general population (26.5 \%) [9]. Smoking prevalence among healthcare professionals in this study was lower in comparison to results from other neighboring countries such as Greece [7, 19] (39 \% among physicians and $46 \%$ among nurses, respectively) and Turkey [14, 20] (38\% among physicians and $45 \%$ among nurses, respectively). An encouraging finding was that the majority of the participants were classified as having low nicotine dependence $(61.3 \%)$, and $71.8 \%$ of current smokers expressed the wish to quit.

Several countries have achieved a considerable decline in tobacco consumption among healthcare professionals in the last decades. In Cyprus however, it is not known whether smoking prevalence among physicians and nurses has increased or declined, since this is the first survey of its kind. According to some of the earliest epidemiological studies in the USA, almost $40 \%$ of physicians smoked in 1959, a finding which has shown remarkable decline, reaching less than $10 \%$ by the mid1990s [14]. Contemporary data reveal that only $2 \%$ of

Table 4 Healthcare professionals' attitude towards counseling patients to quit smoking by smoking status and level of dependence

\begin{tabular}{|c|c|c|c|c|c|c|c|}
\hline & \multicolumn{2}{|c|}{ Rarely/Never } & \multicolumn{2}{|c|}{ Sometimes } & \multicolumn{2}{|c|}{ Often } & \multirow[b]{2}{*}{$p$ value } \\
\hline & $\overline{\mathrm{N}}$ & $\%$ & $\overline{\mathrm{N}}$ & $\%$ & $\bar{N}$ & $\%$ & \\
\hline \multicolumn{8}{|l|}{ Smoking Status } \\
\hline Never & 3 & 1.2 & 6 & 2.4 & 244 & 96.4 & \multirow[t]{3}{*}{$<0.001$} \\
\hline Current & 16 & 11.5 & 22 & 15.8 & 101 & 72.7 & \\
\hline Former & 3 & 11.5 & 1 & 3.8 & 22 & 84.6 & \\
\hline \multicolumn{8}{|l|}{ FTQ-Level of Dependence } \\
\hline Low nicotine dependence & 7 & 8.1 & 12 & 14.0 & 67 & 77.9 & \multirow[t]{3}{*}{0.131} \\
\hline Medium nicotine dependence & 5 & 13.2 & 8 & 21.1 & 25 & 65.8 & \\
\hline High nicotine dependence & 4 & 30.8 & 2 & 15.4 & 7 & 53.8 & \\
\hline
\end{tabular}


American physicians smoke [14]. A similar downward trend was also seen in the UK, where smoking rates among British doctors fell from 62 to 18 \% during the period 1951-1990 [21]. A survey performed in 2011 indicated that only $7 \%$ of doctors were current smokers, which is a significantly lower rate than in the general population (24\%) [22]. On the contrary, $45 \%$ of Chinese and $43 \%$ of Japanese physicians respectively, were identified as current smokers [12]. Similar results were also reported for Kuwait (38 \%), Estonia (24.9 \% for males and $10.8 \%$ for female doctors) and France (34 \%) [14]. Smoking rates have also declined among nurses during the last decades, this decline however, was of a smaller magnitude compared to doctors whereas, in some countries the percentage of nurses who smoke is still very high [8]. Findings from Spanish and Italian studies reported high smoking prevalence among nurses $(28 \%$ [23] and $41 \%$ [20, 24] respectively), while according to a survey in Britain, in 2011, nurses were more likely to be current smokers (8.7\%) than doctors (7\%) [22].

Results of the present study showed that gender, age, marital status and family history of smoking were significantly and independently associated with smoking status, whereas occupation (physician vs. nurse) was not found to be associated with smoking status. This is consistent with results of other studies that showed that male and unmarried physicians $[6,7,11,25]$ and nurses [26], with a family history of tobacco use, were more likely to become smokers. Both physicians and nurses reported that their main motive to quit smoking was health problems, which is consistent with results from other studies $[6,7,11,25]$. Nearly all surveys that have studied the smoking habits of healthcare professionals, showed that a high percentage had made at least one attempt for smoking cessation [7, 11, 19, 27, 28]. In this study, about half of physicians (48.5 \%) and nurses (51.9 \%) who were smokers had attempted to quit smoking in the past. An interesting finding was that physicians reported a significantly higher success rate compared to nurses (62.5\% vs. $31.5 \%)$. Nevertheless, only $6.5 \%$ of the participants used smoking cessation programs in their attempt to quit smoking questioning thus the availability and usefulness of such programs in Cyprus.

Other studies have suggested that the majority of physicians and nurses agree that their smoking behavior possibly influences their attitude towards counseling patients to quit smoking $[19,28,29]$. A study regarding smoking prevalence of qualified nurses in Northern Ireland, found that nurses who continue to smoke were less willing to take on the role of a health promoter towards patients who smoke [30]. This finding is supported by the results of the present study showing that current smokers discuss less often with their patients about smoking cessation interventions, compared to former and never-smokers. In addition, nurses counseled their patients more often than physicians. This may be attributed to the fact that nurses spend more time with the patients and feel that counseling may be part of their responsibilities. A study which was performed in San Francisco in 1990, found that nurse practitioners discussed smoking with patients more often than did physicians ( $64 \%$ vs. $50 \%$; $p<0.01$ ), asked patients more often whether they were interested in quitting ( $49 \%$ vs. $40 \%$; $p<0.01)$, distributed more smoking-cessation literature to patients $(37 \%$ vs. $25 \% ; p<0.01)$ and made more follow-up appointments (36 \% vs. $19 \% ; p<0.01$ ) [31]. In contrast, an Australian study reported that nurses are too busy to discuss with their patients about smoking cessation [26].

Studies in Italian [27] and Chinese [32] populations revealed that chest physicians and healthcare providers respectively, reported lack of formal medical teaching about smoking cessation strategies. Similarly, in BosniaHerzegovina more than half of the healthcare professionals reported that they were inadequately prepared to counsel patients [29]. However, in our study the majority of both physicians and nurses felt confident about their knowledge regarding smoking cessation interventions. This is encouraging, since data suggest that healthcare professionals who felt confident about their knowledge, counsel their patients to quit smoking more often. An encouraging finding of this study was the fact that current smokers were positive towards receiving specialized training for smoking cessation, during their career. However, there was a group of doctors who seem to ignore the harmful properties of smoking and admitted that they do not recommend smoking cessation in situations like lung problems (7.5\%), use of contraception pills (44.8\%), damage in mouth and lips (47.8\%), pregnancy (13\%), nervousness and anorexia (58.2 \%), and insomnia $(59.7 \%)$. This could be attributed to possible knowledge gaps about the destructive effects of smoking or due to their own smoking behaviors. The latter finding is in line with other studies reporting that many healthcare professionals smoke in front of their patients $[7,25,28]$ and agree with the statement that smoking is not as harmful as experts declare [11]. In our study, only a small group of participants reported smoking in front of their patients $(5.9 \%$ of physicians and $8 \%$ of nurses respectively), indicating failure to fulfill their function as role models for their patients and society.

In the context of harmonization of Cypriot legislation with European directives on smoking, the provisions of the law "Protection of Health-Tobacco Control" were applied in 2010. The law specifically prohibits smoking in recreational areas and in enclosed public places. However, the main problem observed is the failure to 
practically apply the legislation in most areas, even in hospitals. Healthcare professionals who are smokers, still smoke even at the hospital setting. So far additional measures to ban smoking have not been taken among healthcare professionals, however the Ministry of Health will proceed with the preparation of Regulations in an effort to improve legislation and enhance the efforts for its implementation [33].

\section{Limitations of the study}

One important limitation of the study is the crosssectional design that only measures the social phenomenon at a specific moment in time and we were thus unable to measure the level of behavioral change concerning smoking habits. Nevertheless, having enough sample size, the aims of the study could be achieved with a cross-sectional design. In addition, smoking prevalence among physicians and nurses is likely to be underestimated, as data were selfreported. Physicians and nurses are perceived as most knowledgeable about the devastating effects of smoking; therefore, they may be prone to self-deception or understatement (misclassification bias). However, the anonymity of the questionnaire supports the possibility of the responses being true. Furthermore, the sample collection was limited to NGH and doctors and nurses from other hospitals in Cyprus did not participate in the study. It is worth noting that the NGH employs almost half of the physicians and nurses that work in the public healthcare sector of Cyprus, and the sample is likely to be representative; nevertheless, one should be cautious about generalizing the findings to all Cypriot physicians and nurses.

\section{Conclusions}

According to the results of this study, smoking prevalence among healthcare professionals was $28.2 \%$ in Cyprus, reflecting the prevalence of the general population. Factors associated with smoking status were age, gender, marital status and family history of smoking. Regarding the attitudes of healthcare professionals towards counseling patients to quit smoking, the study showed that their personal smoking behavior affected their intention to counsel patients to quit smoking. The results of this study support the need for further training of healthcare professionals towards smoking cessation strategies in order to improve their knowledge and consequently their efforts of providing counseling and support to their patients who wish to quit smoking.

\section{Ethics approval and consent to participate}

According to the Cyprus National Bioethics Committee the study does not fall within the competence of the Cyprus National Bioethics Committee for bioethical review.

\section{Abbreviations}

FTQ: Fagerstrom Nicotine Tolerance Questionnaire; NGH: Nicosia General Hospital; WHO: World Health Organization.

\section{Competing interests}

The authors declare that they have no competing interests.

\section{Authors' contributions}

SZI and AG conceived the study and participated in its design along with $T Z$, SZ and AP. Data acquisition was performed by AG. Data was analyzed by SZI and SZ and interpreted by SZI, SZ, TZ and AP. SZI drafted the manuscript, which was revised by $T Z, S Z, A P$, and AG. All authors read and approved the final manuscript.

\section{Acknowledgements}

The authors would like to thank the pulmonologists and administrative personnel of the Respiratory Department Clinic of the NGH, for their help with data collection.

\section{Author details}

${ }^{1}$ Health Center of University of Cyprus, Cyprus International Institute for Environmental and Public Health in association with the Harvard T.H. Chan School of Public Health, Cyprus University of Technology, 1, Panepistimiou Avenue, 2109 Aglantzia, Nicosia, Cyprus. ${ }^{2}$ Open University of Cyprus, 33 Giannou Kranidioti Avenue, 2220 Latsia, Nicosia, Cyprus. ${ }^{3}$ Cyprus International Institute for Environmental and Public Health in association with the Harvard T.H. Chan School of Public Health, Cyprus University of Technology, 95 Eirinis Street, 3041 Limassol, Cyprus. ${ }^{4}$ Respiratory Department Clinic, Nicosia General Hospital, 215 Nicosia - Limassol Old Road, 2029 Strovolos, Nicosia, Cyprus.

Received: 24 July 2015 Accepted: 31 March 2016

Published online: 07 April 2016

\section{References}

1. World Health Organization. Tobacco. Key Facts. 2014 [cited 2014 December 16]; Available from: http://goo.gl/o05e9F. Accessed 16 Dec 2014.

2. Jamison, Dean $\mathrm{T}$, et al, Editors. Disease control priorities in developing countries. Washington: World Bank Publications; 2006.

3. SA. Chapter 1 Burden: mortality, morbidity and risk factors. 2008 [cited 2013 December 16]; Available from: http://goo.gl/KIlwaE. Accessed 16 Dec 2013.

4. Hecht SS. Cigarette smoking and lung cancer: chemical mechanisms and approaches to prevention. Lancet Oncol. 2002;3(8):461-9.

5. Jason L. Nicotine addiction and smoking cessation treatments. Adv Psychiatr Treat. 2002:8:42-8.

6. Parna K, et al. Comparison of knowledge, attitudes and behaviour regarding smoking among Estonian and Finnish physicians. Soz Praventivmed. 2005; 50(6):378-88

7. Sotiropoulos A, et al. Smoking habits and associated factors among Greek physicians. Public Health. 2007;121(5):333-40.

8. Hillier S. Nurses' smoking habits. Postgrad Med J. 1973;49(576):693-4.

9. European Health Servey 2008. Cyprus. Nicosia: Statistical Service; 2008. p. 1-258.

10. Man MA, et al. Why do doctors smoke? Pneumologia. 2009;58(3):195-200.

11. Parna K, Rahu K, Rahu M. Smoking habits and attitudes towards smoking among Estonian physicians. Public Health. 2005;119(5):390-9.

12. Pipe A, Sorensen M, Reid R. Physician smoking status, attitudes toward smoking, and cessation advice to patients: an international survey. Patient Educ Couns. 2009:74(1):118-23.

13. Shkedy $Y$, Feinmesser RM, Mizrachi A. Smoking habits among Israeli hospital doctors: a survey and historical review. Isr Med Assoc J. 2013;15(7):339-41.

14. Smith DR, Leggat PA. An international review of tobacco smoking in the medical profession: 1974-2004. BMC Public Health. 2007;7:115.

15. 2008 Annual Report of the Ministry of Health, Cyprus. 2009 [cited 2013 August 10]; Available from: http://www.moh.gov.cy/moh/moh.nsf/page57 gr/page57_gr?OpenDocument. Accessed 10 Aug 2013

16. 2010 Annual Report of the Ministry of Health, Cyprus. 2011 [cited 2013 August 10]; Available from: http://goo.gl/gMCASm. Accessed 10 Aug 2013.

17. Heatherton TF, et al. The fagerstrom test for nicotine dependence: a revision of the fagerstrom tolerance questionnaire. $\mathrm{Br} J$ Addict. 1991;86(9):1119-27. 
18. Centers for Disease Control and Prevention. Disability and Health Data System. Data Guite-Health Topics. 2013 [cited 8 Dec 2013]; Available from: http://dhds.cdc.gov/guides/healthtopics/indicator?i=smokingstatus.

19. Beletsioti-Stika P, Scriven A. Smoking among Greek nurses and their readiness to quit. Int Nurs Rev. 2006;53(2):150-6.

20. Sezer H, Guler N, Sezer RE. Smoking among nurses in Turkey: comparison with other countries. J Health Popul Nutr. 2007;25(1):107-11.

21. Doll $R$, et al. Mortality from cancer in relation to smoking: 50 years observations on British doctors. Br J Cancer. 2005;92(3):426-9.

22. Sela BA. Time for setting a good example: physicians, quit smoking now. Isr Med Assoc J. 2013;15(7):379-81.

23. Ruiz-Canela M, Martínez-González MA, López-del Burgo C, de Irala J, Beunza JJ, Bes-Rastrollo M. Are smoking habits changing among spanish health professionals? Results from the SUN cohort 1999-2008. Tobacco Use Insights. 2009;2:17-24.

24. Zanetti $F$ et al. Smoking habits, exposure to passive smoking and attitudes to a non-smoking policy among hospital staff. Public Health. 1998;112(1):57-62.

25. Mahfouz AA et al. Tobacco use among health care workers in southwestern Saudi Arabia. Biomed Res Int. 2013;2013:960292.

26. Nagle A, Margot S, Redman S. Australian nurses'smoking behaviour, knowledge and attitude towards providing smoking cessation care to their patients. Health Promot Int. 1999;14(2):133-44.

27. Nardini $\mathrm{S}$, et al. The influence of personal tobacco smoking on the clinical practice of Italian chest physicians. Eur Respir J. 1998;12(6):1450-3.

28. Perrin PC, Merrill RM, Lindsay GB. Patterns of smoking behavior among physicians in Yerevan, Armenia. BMC Public Health. 2006;6:139.

29. Hodgetts G, Broers T, Godwin M. Smoking behaviour, knowledge and attitudes among family medicine physicians and nurses in Bosnia and Herzegovina. BMC Fam Pract. 2004;5:12.

30. McKenna $\mathrm{H}$ et al. Qualified nurses' smoking prevalence: their reasons for smoking and desire to quit. J Adv Nurs. 2001;35(5):769-75.

31. Zahnd EG, et al. Counseling medical patients about cigarette smoking: a comparison of the impact of training on nurse practitioners and physicians. Nurse Pract. 1990;15(3):10-3. 17-8.

32. Yan J, et al. Smoking behavior, knowledge, attitudes and practice among health care providers in Changsha city, China. Nicotine Tob Res. 2008;10(4):737-44.

33. National strategy for control of smoking in Cyprus. 2012 [cited 2015 December 30]; Available from: http://goo.gl/4jjXhG. Accessed 30 Dec 2015 .

\section{Submit your next manuscript to BioMed Central and we will help you at every step:}

- We accept pre-submission inquiries

- Our selector tool helps you to find the most relevant journal

- We provide round the clock customer support

- Convenient online submission

- Thorough peer review

- Inclusion in PubMed and all major indexing services

- Maximum visibility for your research

Submit your manuscript at www.biomedcentral.com/submit

) Biomed Central 